\title{
Robust Watermarking Scheme Applied to Radiological Medical
}

\section{Images}

\section{Raul RODRIGUEZ COLIN ${ }^{\dagger a)}$, Student Member, Claudia FEREGRINO URIBE ${ }^{\dagger b)}$, and Jose-Alberto MARTINEZ VILLANUEVA ${ }^{\dagger c)}$, Nonmembers}

SUMMARY We present a watermarking scheme that combines data compression and encryption in application to radiological medical images. In this approach we combine the image moment theory and image homogeneity in order to recover the watermark after a geometrical distortion. Image quality is measured with metrics used in image processing, such as PSNR and MSE.

key words: watermarking, data hiding, medical images

\section{Introduction}

Digital image watermarking has been proposed in the literature as a method to enhance medical data security, confidentiality and integrity, especially for data regarding patient information (i.e., personal data, studies and diagnosis) [1]. Medical image watermarking requires extreme care when embedding additional data in the medical images because the additional information must not affect the image quality to such an extent that this information could change the diagnosis. The aim of watermarking in the medical field is to invisibly embed a message in an image [2]. Recent modalities of Computed Radiology, Magnetic Resonance and Computed Tomography obtain digital images in DI$\mathrm{COM}$ format (standard for Digital Imaging and Communications in Medicine), store patient data, and study information and images. Given the increasing use of Internet in medicine, the need for security and the importance of the DICOM standard, we propose the use of DICOM metadata as a watermark to embed in medical images extracted from the DICOM file and converted to JPEG lossless format. By encrypting the message to be hidden, the transmission of medical images will be more secure. We have used the stream cipher RC4 [3].

\section{Proposed Scheme}

In this work we propose a blind watermarking scheme divided into two stages. The first stage consists in constructing the watermark as follows: 1) Generate the watermark using the DICOM file, 2) Compress the DICOM data in

Manuscript received July 4, 2007.

Manuscript revised September 5, 2007.

The authors are with the National Institute of Astrophysics, Optics and Electronics, Luis Enrique Erro No. 1 Sta. Maria Tonantzintla, Puebla, Mexico C.P. 72840.

a)E-mail: raulrc@inaoep.mx

b) E-mail: cferegrino@inaoep.mx

c) E-mail: jamartinez@ inaoep.mx

DOI: 10.1093/ietisy/e91-d.3.862

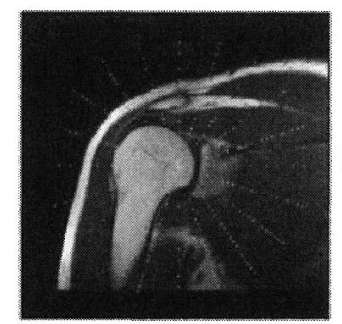

POSSIBLE EMBEDDING POSITION5

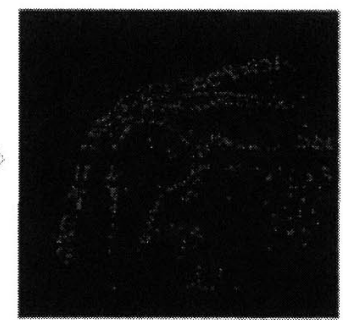

FINAL POSITIONS
Fig. 1 Process to select pixels.

order to reduce the amount of data to embed (we apply a Huffman compression), and 3) Cipher the compressed data to enhance the security of the original message using RC4 scheme. The second stage consists in embedding the watermark into the image in JPEG lossless format. This format is useful to fairly compare the results with other watermarking methods that use 8 bits JPEG gray scale images. However, the method can be adapted to work with any bit sizes and formats. Figure 1 shows the process of selecting the pixels where the data will be embedded. The complete process is as follows:

1. The image is scanned by applying a polar mapping and by using the centroid of the image as the origin of this scan. To obtain the centroid, we used the image moment theory [4], [5] in order to correct the geometric distortions before extracting the watermark.

2. For each pixel in polar form, the homogeneity is calculated using the variance $\left(\sigma^{2}\right)$ of a block of $k \times k$ pixels, where $k$ is the size of the window. If $\sigma^{2} \geq T h$ then the pixel is selected to embed data in this position according to formulas (1) and (2). Th is a threshold for the homogeneity, and both $T h$ and $k$ are given by the user.

$$
\begin{aligned}
& \sigma^{2}=\frac{1}{k k} \sum_{x=0}^{k-1} \sum_{y=0}^{k-1}(f(x, y)-\mu)^{2} \\
& \mu=\frac{1}{k k} \sum_{x=0}^{k-1} \sum_{y=0}^{k-1} f(x, y)
\end{aligned}
$$

The embedding process is described below:

1. A block of size $(k \times k)$ is obtained with its center in the position of each of the selected pixels 
2. a) If the bit to embed is " 1 ", then change the luminance value of the central pixel to make sure that:

$$
L_{\text {real }} \geq L_{\text {mean }}+\delta_{1}
$$

b) If the bit to embed is " 0 ", then change the luminance value of the pixel to make sure that:

$$
L_{\text {real }}<L_{\text {mean }}-\delta_{2}
$$

where $\delta_{1}$ and $\delta_{2}$ are calculated based on the homogeneity and luminance of the block $(k \times k), L_{\text {real }}$ is the gray-scale level of the pixel, and $L_{\text {mean }}$ is the gray-scale level mean of the block [6]. The extraction process is the following:

1. Locate the changed pixel by using the polar mapping and by starting from the centroid of the image.

2. The decision threshold to extract the watermark is:

- If $L_{\text {real }} \geq L_{\text {mean }}$ then the extracted bit is " 1 ".

- If $L_{\text {real }}<L_{\text {mean }}$ then the extracted bit is " 0 ".

The values of $\delta_{1}$ and $\delta_{2}$ are not required to extract the watermark.

\section{Experimental Results}

The experiments were carried out in three DICOM files shown in Fig. 2. where a) and b) are $256 \times 256 \times 16$ bits, and c) $1024 \times 1024 \times 12$ bits gray scale medical images. The three images were resized to $512 \times 512 \times 8$ bits when they are converted to JPEG lossless format. The homogeneity is calculated using a window of $3 \times 3$ pixels. The watermark consists of the DICOM metadata plus the medical diagnostic of the study, 4800 bits in total. In order to determine the degradation of the watermarked image with respect to the original image, we apply the PSNR and MSE metrics to measure the distortion produced after the embedding process [7], and we apply the NCC to evaluate the similarity between the original watermark and the extracted watermark.

$$
\begin{aligned}
& M S E=\frac{1}{M N} \sum_{x=0}^{M-1} \sum_{y=0}^{N-1}\left(f(x, y)-f^{\prime}(x, y)\right)^{2} \\
& P S N R=10 * \log _{10} \frac{255^{2}}{M S E} d B \\
& N C C=\frac{\sum_{x=0}^{R-1} \sum_{y=0}^{C-1}\left(W(x, y) * W^{\prime}(x, y)\right)}{\sum_{x=0}^{R-1} \sum_{y=0}^{C-1}|W(x, y)|^{2}}
\end{aligned}
$$

In Table 1, the degradation and distortion in the watermarked images are presented. In Fig. 3 one of the images after some attacks such as brightness, contrast, compression and rotation is shown. The percentage of recovered watermark after these attacks is shown in Table 2. From the results it can be seen our proposed scheme is robust to several attacks while allowing to recover the watermark message.

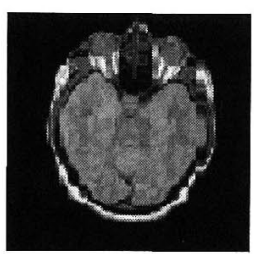

(a) Brain

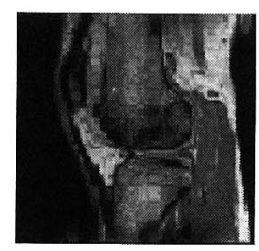

(b) Knee

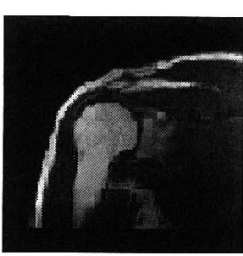

(c) Shoulder
Fig. 2 Images used in the experiments.

Table 1 Imperceptibility using PSNR (dB) and MSE metrics.

\begin{tabular}{|c|c|c|}
\hline Image & PSNR & MSE \\
\hline Brain & 37.1 & 12.5 \\
\hline Knee & 42.3 & 4.2 \\
\hline Shoulder & 41.1 & 5.4 \\
\hline
\end{tabular}

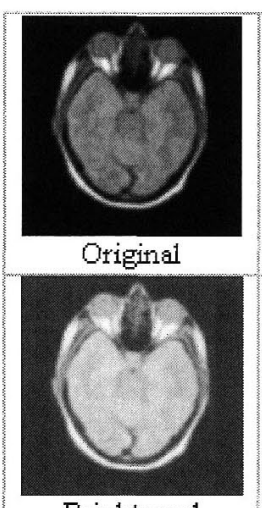

Brightened

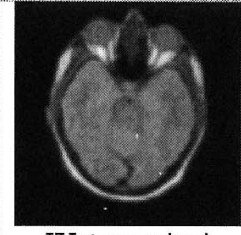

Watermarked

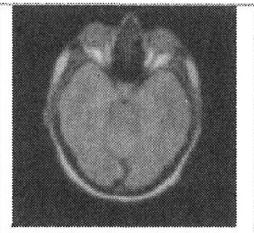

Contrasted

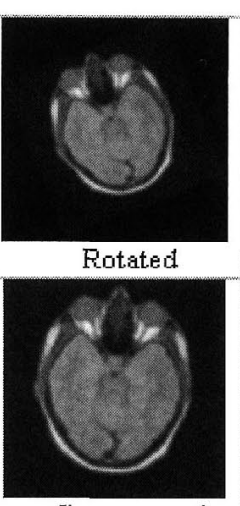

Compressed
Fig. 3 Different attacks in the watermarked image.

Table 2 Recovered data after brightness, contrast, JPEG compression with and 35 degrees rotation.

\begin{tabular}{|c|c|c|c|c|}
\hline Image & Brightness & Contrast & $\begin{array}{c}\text { JPEG } \\
\text { Compression }\end{array}$ & Rotation \\
\hline Brain & $98.5 \%$ & $97.0 \%$ & $94.5 \%$ & $81.5 \%$ \\
\hline Knee & $99.0 \%$ & $96.0 \%$ & $90.5 \%$ & $83.4 \%$ \\
\hline Shoulder & $97.0 \%$ & $95.5 \%$ & $91.5 \%$ & $80.3 \%$ \\
\hline
\end{tabular}

\section{Conclusions}

We have presented a blind watermarking scheme applied to medical images that has shown to be robust to several attacks. Additionally, the watermark has been compressed with the Huffman algorithm and ciphered with RC4 in order to diminish the message size and to add security to the whole system. The algorithm is robust by both embedding and extracting the watermark message. Additionally, using the homogeneity allows us to obtain a better accuracy in the extraction process than by using a simple LSB method.

\section{References}

[1] G. Coatrieux, H. Maitre, B. Sankur, Y. Rolland, and R. Collorec, "Relevance of watermarking in medical imaging," IEEE-embs Information Technology Applications in Biomedicine, pp.250-255, 2000. 
[2] W. Puech and J.M. Rodrigues, "A new crypto-watermarking method for medical images safe transfer," Proc. 12th European Signal Processing Conference, pp.1481-1484, 2004.

[3] B. Schneier, Applied Cryptography, 2nd ed., John Wiley \& Sons, 1996.

[4] P. Dong, J.G. Brankov, N.P. Galatsanos, Y. Yongyi, and F. Davoine, "Digital watermarking robust to geometric distortions," IEEE Trans. Image Process., vol.14, no.12, pp.2140-2150, 2005.

[5] L. Tung-Lam and N. Thi-Hoang-Lan, "Digital image watermarking with geometric distortion corrections using the moment image theory," International Conference on Research Innovation and Vision for the Future (RIVF), 2004.

[6] Y. Wang and A. Pearmain, "Blind image data hiding based on self reference," Pattern Recognit. Lett., vol.25, no.15, pp.1681-1689, 2004.

[7] B. Plaintz and A. Maeder, "Medical image watermarking: A study on image degradation," Proc. Australian Pattern Recognition Society, 2005. 